\title{
Consideraciones sobre el origen y primera dispersión del aguacałe (Persea americana, Lauraceae)
}

\section{E. Galindo-Tovar ${ }^{1}$, A. M. Arzate-Fernández ${ }^{2}$}

i Facultad de Ciencias Biológicas y Agropecuarias, Universidad Veracruzana, Apartado posTAl i 77, 45000 Córdoba, Veracruz, MÉxico. Megalindo@Uv.mX

2 Facultad de Ciencias Agrícolas, Universidad Autónoma del Estado de México, Carretera Toluca-Ixtlahuaca KM i 5 , Toluca, Estado de México, México.

\section{ABSTRACT}

The origin of the avocado and its varieties has been placed in the Mesoamerican area; however there are still some aspects that have not been entirely explained. We surveyed this issue integrating geological, archaeological and paleoecological data that allowed us to examine the most ancient ancestors, their geographic distribution and their possible dispersion routes. In the light of these data, we propose that the origin of the avocado could have been in the area currently occupied by Sierra Nevada (California) and that it could have occurred when the mountains emerged about 8 to 5 million years ago. Afterwards, the avocado migrated south where different domestications occurred and the current varieties evolved. Each variety was adapted to different ecological conditions and became domesticated by different cultural groups.

\section{KEY WORDS:}

Mesoamerica, dispersion, wild avocados.

El aguacate (Persea americana Mill.), pertenece a la familia Lauraceae, uno de los grupos de angiospermas más antiguos (RENNER, 1999).

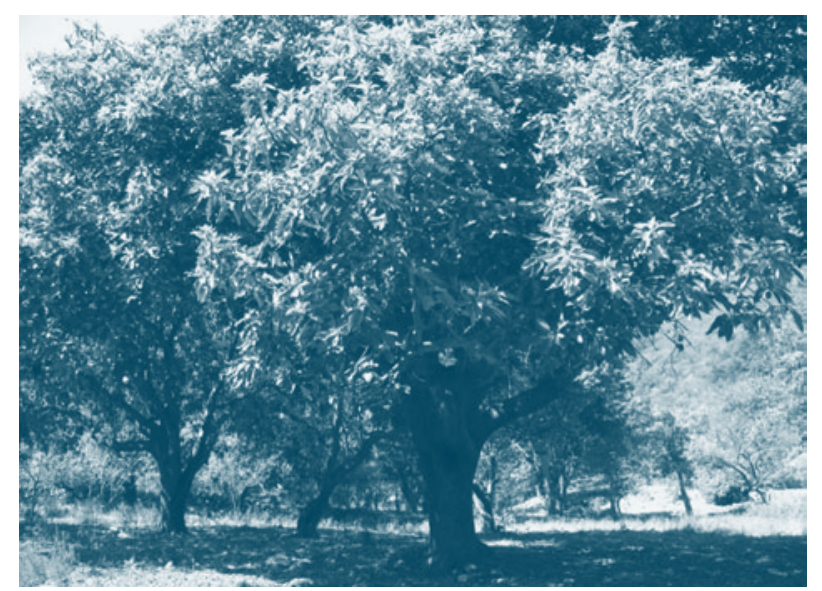

Figura 1: Persea americana Mill 
Se caracteriza por su gran variabilidad y por ser una de las familias del reino vegetal más numerosas, con alrededor de 92 géneros descritos y un número indeterminado de especies que varía de 2.840 a 3.340 que se distribuyen por todas las regiones tropicales y subtropicales del mundo (RENNER, 2004).

Para esta especie se han descrito ocho variedades, de las cuales tres son ampliamente conocidas como razas hortícolas: la Mexicana (Persea americana var. drymifolia [Schltdl. et Cham] S.F. Blake), la Guatemalteca (Persea americana var. guatemalensis [L.O. Williams] Scora) y la Antillana (Persea americana. var. americana) (CHANDERBALI et al., 2008). Estas variedades y su hibridación han dado origen a los cultivares modernos del aguacate (KNIGHT \& CAMPBELL, 1999).

La secuenciación de cuatro loci indica diferencias en la composición de los haplotipos de estas tres variedades, lo que sugiere que diferentes poblaciones de aguacate fueron separadas por largos periodos de tiempo. Una vez que el hombre empezó a utilizar este fruto, se inició una amplia diseminación y su domesticación; lo que dio origen a los diferentes tipos de aguacate que actualmente conocemos (CHEN et al., 2009).

La relación del aguacate con el hombre ha sido documentada con datos paleohistóricos reportados por algunos cronistas de la Nueva España (COBO, [1653] 1964; ACOSTA, [1590] 1985; SAHAGÚN, [1590] 2002; BENAVENTE, [1542] 2003). Estos datos muestran que las tres razas hortícolas del aguacate tienen profundas raíces históricas desde tiempos precolombinos en Mesoamérica, área donde según GALINDO et al. (2008) ocurrió su domesticación. Sin embargo, aún no está claro dónde y cuándo se originó el aguacate. El principal problema es que aún no se conoce el prototipo silvestre; probablemente debido al hecho de que se ha extinguido, o ha cambiado a través de su evolución; ya que durante su proceso de domesticación, ha sido profundamente modificado, por lo que es difícil distinguir entre árboles domesticados y silvestres.

Son varios los centros de origen que han sido propuestos para esta especie. Por ejemplo, POPENOE (1935) propuso que el centro de origen del aguacate se debía buscar en el área que va de México al norte de Sudamérica; más tarde, KOPP (1966) y STOREY et al. (1986) propusieron, con base en la gran diversidad actual y la presencia de probables aguacates silvestres, al área de Chiapas (México)Guatemala-Honduras como centro de origen para esta especie y posteriormente, BERGH (1992), propuso el área centro sur de México y Guatemala. Sin embargo, las evidencias son circunstanciales y se fundamentan principalmente en la distribución geográfica actual de la especie; por lo que ha sido difícil esclarecer donde y cuando pudo haberse originado esta especie.

Hasta la fecha, el origen del aguacate se ha buscado en áreas en las que actualmente existen poblaciones con gran diversidad o se han encontrado aguacates con características que son consideradas como silvestres (KOPP, 1966; STOREY et al., 1986; BERGH, 1992). Pero, se ha ignorado la relación de esta especie con datos paleoclimáticos. Además, no se ha relacionado la ubicación de los fósiles con la trayectoria del reemplazo biótico y las respuestas a las catástrofes ambientales, datos que de acuerdo a FIELD (2002) ofrecen información que no debe ser ignorada. Además, tampoco se han incluido datos del pasado que nos informan de la distribución histórica y de respuestas ecológicas, que de acuerdo a MILLAR (1996) no se pueden inferir a partir de la dinámica actual. Todo esto, hace imperante la necesidad de realizar una revisión que permita clarificar el origen de este importante árbol tropical.

Nuestro propósito es reconstruir la historia del aguacate integrando datos geológicos, arqueológicos y paleoecológicos para contribuir a esclarecer su origen.

El origen de los ancestros de la familia Lauraceae se ha ubicado en Gondwana y su dispersión hasta América del Norte ha sido revisada por SCORA \& BERGH (1992). Ellos proponen que el aguacate evolucionó durante el terciario, de progenitores provenientes de Laurasia; y que la especie se originó en América Central, durante el Mioceno-Plioceno (221.8 millones de años) cuando se formó el istmo de Panáma y emergieron las montańas. Sin embargo, de acuerdo a GRAHAM (1998), durante aquel periodo, los bosques lluviosos tropicales no existían en el área de América Central o estaban muy pobremente representados. Además, los datos paleoecológicos 
reportados por PIPERNO et al. (2006), indican que aún en el Cuaternario, a finales del Pleistoceno (12.000 a 9.200 a.C.), los ambientes de las tierras bajas neotropicales, de Guatemala al Amazonas, eran fríos y secos y no fue sino hasta que la temperatura y precipitación se elevaron, que los bosques tropicales reemplazaron a la flora herbácea adaptada a los climas fríos, que dominaban aquellas regiones. Por lo anteriormente expuesto, es poco probable que el aguacate se originara durante el Mioceno-Plioceno en Centroamérica.

Por otro lado, SCORA \& BERGH (1992) documentaron que el género Persea, durante el Eoceno (56-35 millones de años), ya se encontraba en Norteamérica; además, datos paleoecológicos indican que al final del Terciario, el área que hoy ocupa la Sierra Nevada (California) era un lugar de clima subtropical con gran diversidad vegetal en la que los aguacates antiguos eran comunes (MILLAR, 1996). Esta información es complementada con los datos arqueológicos recopilados por SCHRÖEDER (1968), quién reportó la presencia de plantas parecidas al aguacate en la parte norte y centro de California, que datan aproximadamente entre 10 y 60 millones de ańos y de árboles similares al aguacates en el Eoceno, probablemente de hace 50 millones de años. En su reporte, SCHRÖEDER (1968) comenta que estos árboles eran representativos de la flora en varias áreas de California; con un $68 \%$ del total de los especimenes preservados en los fósiles encontrados. Estos datos coinciden con la historia de la vegetación del Terciario que realizó MILLAR (1996). Además, dicho autor reporta que durante el Terciario temprano, la región de la Sierra Nevada era plana y con condiciones de clima subtropical a tropical (cálido-húmedo) apropiadas para el desarrollo de estos árboles. Desde esta perspectiva, es posible que cuando se formó la Sierra Nevada en el Cenozoico tardío (LIU \& SHEN, 1998; WAKABAYASHI \& SAWER, 2001) hace cinco a ocho millones de años, por adaptación se originaran los primeros aguacates modernos en esta área; y no en la zona de Chiapas (México)-Guatemala-Honduras, donde como ya fue comentado, no existían las condiciones apropiadas durante el Mioceno-Plioceno.

Debido a la última glaciación (hace 15.000 años), ocurrida en la Sierra Nevada el clima cambió a seco y frío, ocasionando que los aguacates se desplazaran hacia el sur y se extinguieran en esta área, dejando como única evidencia los fósiles foliares reportados por SCHRÖEDER (1986).

La presencia de aguacates silvestres en la Sierra de Nuevo León (STOREY et al., 1986), que de acuerdo a GUTÍERREZ (2009) muestran características contrastantes a las de las variedades nativas que se cultivan en huertas familiares y traspatio, indica una ruta de migración hacia el sur; y el cambio climático explica porqué en el área de origen, actualmente no hay poblaciones naturales de aguacate y también, porqué no se ha encontrado el prototipo silvestre de $P$. americana.

La migración de la semilla de los árboles de aguacate hacia el sur debe haberse realizado con la ayuda de los grandes mamíferos y aves que habitaban el área en esa época (BERGH, 1992; BAELOW, 2002). La diseminación por animales explica el amplia y variable área de dispersión, debida al transporte de las semillas hacia lugares lejanos del centro de origen, donde el clima fue propicio para su desarrollo. Esta migración explica la capacidad de los árboles de aguacate para adaptarse a diversas condiciones; ya que la migración de norte a sur favorece el desarrollo de cierta variabilidad conveniente para la evolución y domesticación.

Según BUCKLER et al.,(1998), el clima en la parte central de México, desde la máxima glaciación hasta 10.000 a.C. era más frío y húmedo que en la actualidad y en el Valle de Tehuacán, el clima era húmedo y hospitalario para el aguacate. Lo anterior sugiere que el aguacate se adaptó a este clima, dónde encontró el ambiente apropiado para su desarrollo y proliferó desde antes de la llegada del hombre. Cuando el hombre llegó a Mesoamérica (hace alrededor de 15.000 años) (GOEBEL et al., 2008) se inició la interacción hombre-planta que dio como resultado el inicio de la domesticación del aguacate. De acuerdo a MACNEISH (1964) y SMITH (1964, 1966), desde hace aproximadamente 10.000 años los primeros pobladores del valle de Tehuacán ya consumían el aguacate y seleccionaban los frutos más grandes. 


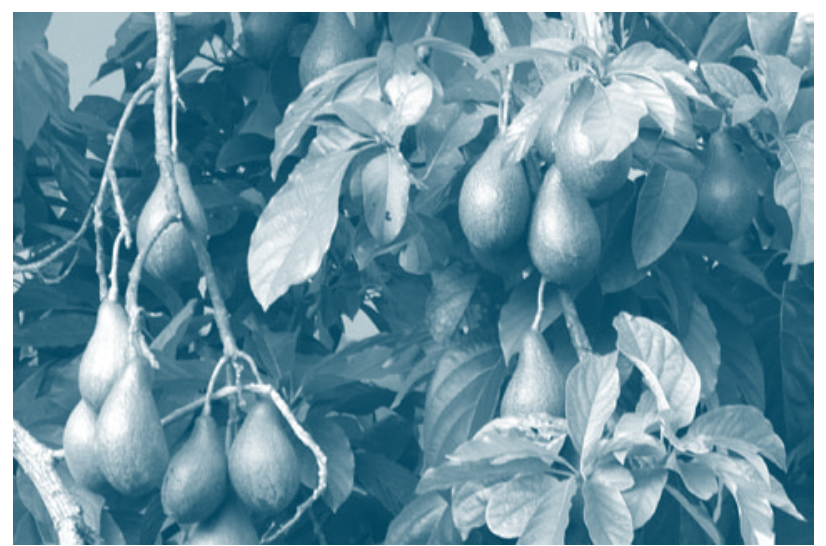

Figura 2: Fruto de Persea americana

Posteriormente, la migración humana y los intercambios comerciales y culturales que se realizaron desde tiempos antiguos entre los diferentes grupos que habitaron desde Mesoamérica hasta el norte de Sudamérica, propiciaron su diseminación y diferentes domesticaciones. En tiempos más recientes la mezcla de estos domesticados fue la base de muchos de los modernos cultivares.

Como resultado de esta investigación se sugiere que cuando se realicen estudios sobre el origen y dispersión de las especies, se integren datos geológicos, paleoecológicos y arqueológicos. En el caso del aguacate, es necesario continuar con este tipo de estudios utilizando las herramientas que actualmente proporciona la genética molecular y determinar las relaciones filogeográficas entre los árboles de aguacate modernos.

\section{BIBLIOGRAFÍA}

ACOSTA J. DE. [1590] 1985. Historia natural y moral de las indias. Fondo de Cultura Económica. México. 444 pp.

BARLOW C. 2002. The ghosts of evolution. Perseus Books Group. Estados Unidos de Norteamérica. 291 pp.

BENAVENTE F. T. M. DE. [1542] 2003. Historia de los indios de la Nueva España. Dastin, S.L. España. 331 pp.

BERGH B. O. 1992. The origin, nature and genetic improvement of avocado. Calif. Avocado Soc. Yearb. 76: 61-75.

BUCKLER IV E. S., D. M. PEARSALL \& T. P. HOLTSFORD. 1998. Climate, plant ecology, and central mexican archaic subsistence. Curr. Anthropol. 39: 152-164.
Chanderbali A. S., V. A. Albert, V. E. T. M. ASHWORTH, M. T. CLEGG, R. E. LITZ, D. E. SOLTIS \& P. S. SOLTIS. 2008. Persea americana (avocado): bringing ancient flowers to fruit in the genomics era. BioEssays 30: 386-396.

CHEN H., P. L. MORELL, V. E. T. M. ASHWORTH, M. DE LA CRUZ \& M. T. CLEGG. 2009. Tracing the geographic origins of mayor avocado cultivars. J. Hered. 100: 56-65.

COBO P. B. [1653] 1964. Historia de Nuevo Mundo. Biblioteca de Autores Españoles. España. 431 pp.

FIELD T. S. 2002. Plant evolution viewed through a functional and paleoclimatic prism. Am. J. Bot. 89: 2034-2036.

GALINDO-TOVAR M. E., N. OGATA-AGUILAR \& A. M. ARZATE-FERNÁNDEZ. 2008. Some aspects of avocado (Persea americana Mill.) diversity and domestication in Mesoamerica. Genet. Resour. Crop Evol. 55: 441-150.

GOEBEL T., M. R. WATERS \& D. H. O'ROURKE. 2008. The late Pleistocene dispersal of modern humans in the Americas. Science. 319: 1497-1502.

GRAHAM A. 1995. Development of affinities between Mexican/Central American and Nothern South American lowland and lower montane vegetation during the tertiary. p. 11-22. In S. P. Churchill et al. (eds.). Biodiversity and Conservation of Neotropical Montane Forests. The New York Botanical Garden. Nueva York, Estados Unidos de Norteamérica.

GUTIERREZ-DIEZ A., J. MARTÍNEZ-DE LA CERDA, E. A. GARCÍA-ZAMBRANO, L. IRACHETA-DONJUAN, J. D. OCAMPO-MORALES \& I. M. CERDAHURTADO. 2009. Estudio de la diversidad genética del aguacate nativo en Nuevo León, México. Rev. Fitotec. Mex. 32: 9-18.

KNIGHT R. J. JR. \& C. W. CAMPBELL. 1999. Ecological adaptation and the evolution of modern avocado cultivars. Rev. Chapingo S. Hort. 5: 49-54.

KOPP L. E. 1966. A taxonomic revision of the genus Persea in the western hemisphere (Persea-Lauraceae). Mem. NY. Bot. Gard. 14: 1-120.

LIU M. \& Y. SHEN. 1998. Sierra Nevada uplift; a ductile link to mantle upwelling under the basin and range province. Geology 26: 299-302.

MACNEISH R. S. 1964. Ancient Mesoamerican civilization. Science. 143: 531-537.

MILLAR C. I. 1996. Tertiary vegetation history. En: Sierra Nevada Ecosystem Proyect: Final report to Congress. University of California. Albania, CA. Estados Unidos de Norteamérica. Pp. 71-122. 
PIPERNO D. R. 2006. Quaternary environmental history and agricultural impact on vegetation in Central America. Ann. Mo. Bot. Gard. 93: 274-296.

POPENOE W. 1935. Origin of the cultivated races of avocados. Calif. Avocado Ass. 1935 Yearb. 19: 184-194.

RENNER S. 1999. Circumscription and phylogeny of Laurales: evidence from molecular and morphological data. Am. J. Bot. 86: 1301-1315.

RENNER S. 2004. Variation in diversity among Laurales, Early Cretaceous to present. Biol. Skr. 55: 441-458.

SAHAGÚN F. B. [1590] 2002. Historia general de las cosas de la Nueva España. Cien de México. México. 1450 pp.

SCHRÖEDER C. A. 1968. Prehistoric avocados in California. Calif. Avocado Soc. 1968 Yearb. 52: 29-34.
SCORA R. W. \& B. O. BERGH. 1992. Origin of the taxonomic relationships within the genus Persea. Proceedings of the Second World Avocado Congress 505-514.

SMITH C. E. J. 1966. Archaeological evidence for selection in avocado. Econ. Bot. 20: 169-175.

SMITH C. E. J. 1969. Additional notes on pre-conquest avocados in Mexico. Econ. Bot. 23: 135-140.

STOREY W. B., B. BERGH \& G. A. ZENTMYER. 1986. The origin, Indigenous range, and dissemination of the avocado. Calif. Avocado Soc. 1986 Yearb. 70: 127-133.

WAKABAYASHI J. \& T. L. SAWYER. 2001. Stream incision, tectonics, uplift, and evolution of topography of the Sierra Nevada, California. J. Geology 109: 539-562. 